Anaesthesist 2022 $\cdot 71: 303-306$ https://doi.org/10.1007/s00101-021-01072-w Eingegangen: 16. April 2021 Überarbeitet: 20. Oktober 2021 Angenommen: 23. Oktober 2021 Online publiziert: 22. November 2021 (c) Der/die Autor(en) 2021

\section{Entwicklung einer Riesenbulla unter Spontanatmung durch „patient self-inflicted lung injury“ bei COVID-19-Pneumonie}

\author{
Nicholas Moellhoff · Philipp Groene' $\cdot$ Ludwig Ney ${ }^{1}$. Daniela Hauer ${ }^{1}$ \\ 'Intensivstation ANIS 5, Klinik für Anaesthesiologie, Klinikum der Universität München, LMU München, \\ München, Deutschland \\ ${ }^{2}$ Abteilung für Hand-, Plastische und Ästhetische Chirurgie, Klinikum der Universität München, LMU \\ München, München, Deutschland
}

Zusammenfassung

SARS-CoV-2 und die damit assoziierte COVID-19-Erkrankung stellen Gesundheitssysteme weltweit vor große Herausforderungen. Fast täglich werden neue Erkenntnisse zu Diagnostik, Klinik und Therapie der Erkrankung publiziert. Dieser Fallbericht beschreibt den letalen Krankheitsverlauf eines 81-jährigen Patienten ohne pulmonale Vorerkrankungen, der als Komplikation der COVID-19-Pneumonie unter nichtinvasiver High-Flow-Sauerstofftherapie eine Riesenbulla entwickelte. Pathophysiologisch kommen/kommt eine virusbedingte diffuse Zerstörung des Alveolargewebes und/oder die "patient self-inflicted lung injury" in Betracht.

\title{
Schlüsselwörter
}

COVID-19 · ARDS · Bulla · High-Flow-Therapie · "Patient self-inflicted lung injury“

\section{Anamnese}

Ein 81-jähriger, normalgewichtiger Mann mit Vorhofflimmern, arterieller Hypertonie und Hypercholesterinämie stellte sich mit seit 9 Tagen bestehender Schwäche und Atemnot in der internistischen Notaufnahme vor. Hustenreiz verneinte der Patient. Er sei Nichtraucher, sportlich aktiv, fahre durchschnittlich über 50 km Fahr$\mathrm{rad} /$ Woche und habe keine pulmonalen Grunderkrankungen. Im Familienkreis gab es mehrere COVID-19-Erkrankungen, bei ihm sei allerdings vor 7 Tagen ein PCRTest auf SARS-CoV-2 negativ ausgefallen.

\section{Befund}

In der Notaufnahme zeigte der Patient eine schwere respiratorische Partialinsuffizienz mit kompensatorischer Hyperventilation $\left(\mathrm{p}_{\mathrm{a}} \mathrm{O}_{2} 56,4 \mathrm{~mm} \mathrm{Hg}\right.$ unter Sauerstoff mit $4 \mathrm{l} / \mathrm{min}, \mathrm{p}_{\mathrm{a}} \mathrm{CO}_{2} 25,8 \mathrm{~mm} \mathrm{Hg}$, Atemfrequenz $22 / \mathrm{min})$. Ein Antigenschnelltest auf SARSCoV-2 war positiv. Eine Aufnahme auf die anästhesiologische Intensivstation wurde zur Überwachung und zur respiratorischen Stabilisierung indiziert.

\section{Diagnose}

\section{Klinische Untersuchung}

Der Patient war bei Aufnahme auf die Intensivstation wach und voll orientiert. Bei Spontanatmung unter Sauersoff mit $6 \mathrm{l} / \mathrm{min}$ über die Nasenbrille zeigte sich eine periphere Sättigung von $93 \%$. Beidseits war ein seitengleich verschärftes Atemgeräusch auskultierbar. Der Patient war normoton, normofrequent und zeigte im EKG die vorbeschriebene Arrhythmia absoluta. Das Abdomen war weich, ohne Druckschmerz und mit regelhaften Darmgeräuschen. Der Patient zeigte eine Spontanmiktion und keine peripheren Ödeme. 

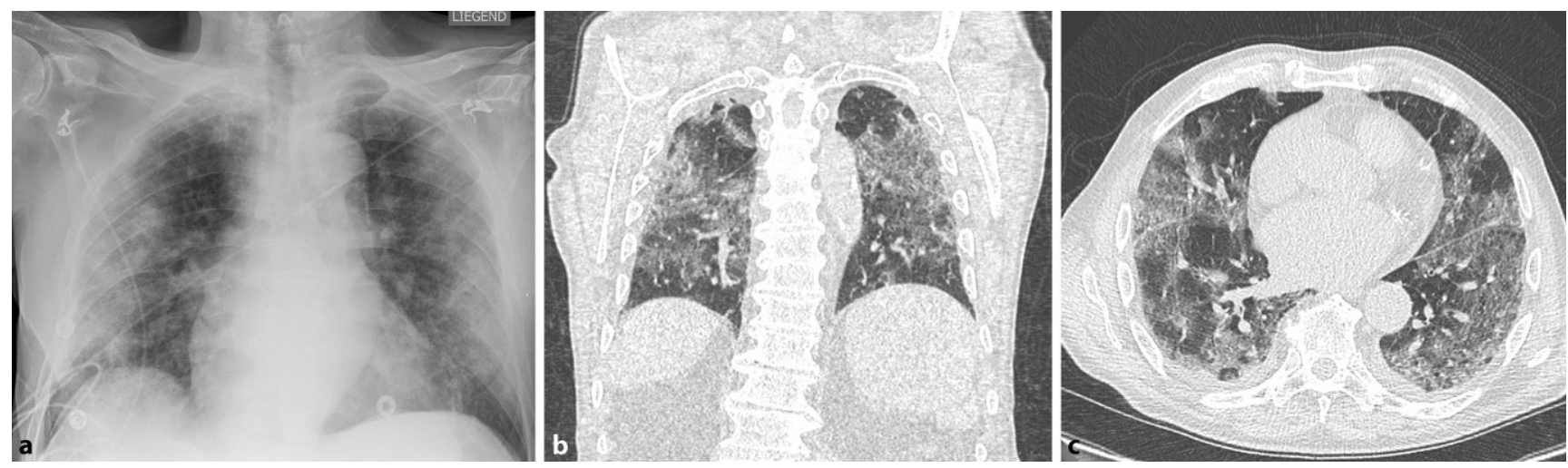

Abb. 1 ॥ Bildgebung bei Aufnahme. a Thoraxröntgenaufnahme mit bipulmonalen grobfleckigen, teils konfluierenden interstitiellen Infiltraten bei COVID-19-Pneumonie. b,c Thorax-CT mit bipulmonalen Konsolidierungen und geografischen Milchglastrübungen mit Prädominanz in den peripheren Lungenabschnitten, vereinbar mit einer COVID-19-Pneumonie
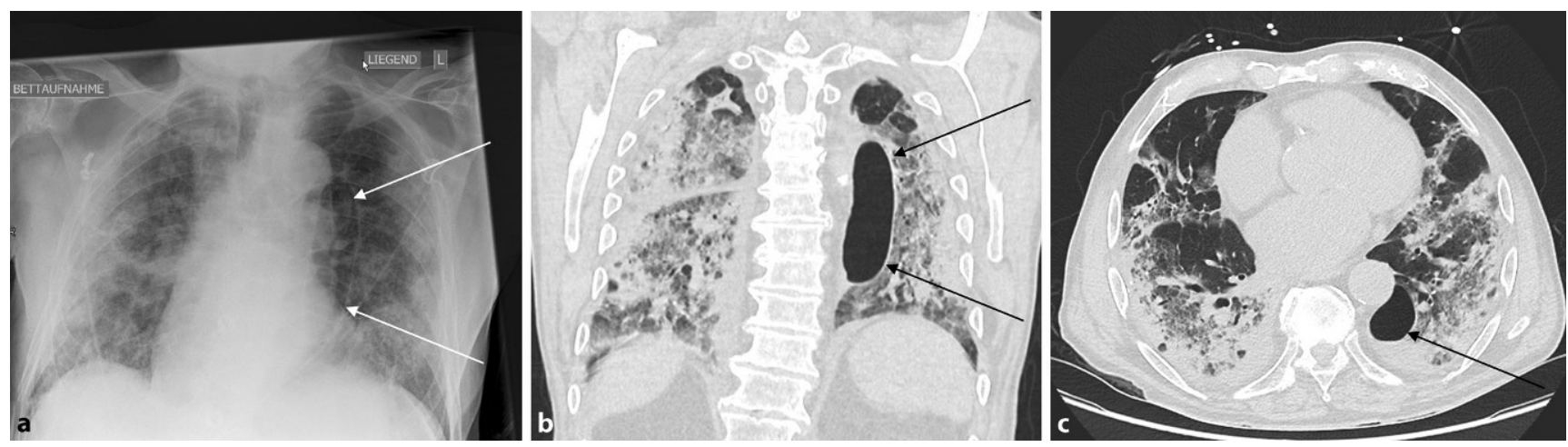

Abb. 2 ム Verlaufskontrolle 7 Tage nach Aufnahme. a Thoraxröntgenaufnahme mit pneumonischen Konsolidierungen bei COVID-19-Pneumonie und ovaler Hypertransparenz paramediastinal links mit V. a. einen ventralseitigen Pneumothorax. b,c Thorax-CT mit bipulmonalen Konsolidierungen sowie Milchglastrübungen bei COVID-19-Pneumonie und Nachweis einer 3,5×3,3 × 10,2 cm großen Bulla links paramediastinal dorsal. Pfeile jeweils auf die Bullae zeigend

\section{Blutgasanalyse, Aufnahmelabor und PCR-Diagnostik}

Die arterielle Blutgasanalyse unter 61 $\mathrm{O}_{2}$ /min ergab: $\mathrm{p}_{\mathrm{a}} \mathrm{O}_{2} 80,6 \mathrm{~mm} \mathrm{Hg} ; \mathrm{p}_{\mathrm{a}} \mathrm{CO}_{2}$ $27,5 \mathrm{~mm} \mathrm{Hg} ; \mathrm{pH} 7,5 ; \mathrm{BE}-0,6 \mathrm{mmol} / \mathrm{l} ;$ Atemfrequenz 21/min.

Das Aufnahmelabor zeigte Veränderungen im Sinne einer ausgeprägten COVID19-Erkrankung: Ferritin: $3928 \mathrm{ng} / \mathrm{ml}$, CRP: 21,8 mg/dl, PCT: $0,3 \mathrm{ng} / \mathrm{ml}, \mathrm{LDH}: 405 \mathrm{U} / \mathrm{l}$, Troponin T: $0,014 \mathrm{ng} / \mathrm{ml}$, Leukozyten: 4,41 G/l, Hb: 13,1 g/dl, Lymphozyten: 0,51 G/l, eosinophile Granulozyten:0,02 G/I, Fibrinogen: $\quad>900 \mathrm{mg} / \mathrm{dl}$, D-Dimer: $1,2 \mu \mathrm{g} / \mathrm{ml}$.

Der PCR-Test auf SARS-CoV-2 bestätigte die Viruserkrankung, zeigte im kombinierten oro-/nasopharyngealen Abstrich mit ca. $7000 \mathrm{Kopien/ml}$ allerdings eine geringe Viruslast.

\section{High-Resolution-Computer- tomographie}

Die CT-Diagnostik zeigte bipulmonale Konsolidierungen sowie Milchglastrübungen, vereinbar mit einer COVID-19Pneumonie (ब Abb. 1).

\section{Therapie und Verlauf}

Einen Tag nach Aufnahme wurde bei fortschreitender Hypoxämie eine HighFlow-Sauerstofftherapie $\left(\mathrm{F}_{1} \mathrm{O}_{2} \quad 50 \mathrm{Vol} . \%\right.$, Flow 40 I/min, darunter: $\mathrm{p}_{\mathrm{a}} \mathrm{O}_{2} 69,1 \mathrm{~mm} \mathrm{Hg}$; $\mathrm{p}_{\mathrm{a}} \mathrm{CO}_{2} 27,4 \mathrm{~mm} \mathrm{Hg} ; \mathrm{pH} 7,5 ; \mathrm{BE}-0,1 \mathrm{mmol} / \mathrm{l}$; Atemfrequenz $19 / \mathrm{min}$; Horowitz-Index $138 \mathrm{~mm} \mathrm{Hg}$ ) initiiert, welche im weiteren Verlauf eskaliert wurde. Dyspnoe wurde überwiegend verneint, es zeigte sich aber bereits unter leichter Belastung (Mobilisation an die Bettkante, Atemgymnastik) ein deutlicher Abfall der Sauerstoffsättigung auf $70-80 \%$. Unter der leitliniengerechten
Therapie mit Dexamethason ( $6 \mathrm{mg} / \mathrm{Tag}$ p.o. für 10 Tage) [8] kam es zu einer endogenen Reaktivierung des Herpes-simplex-Virus, die mit Aciclovir behandelt wurde. Aufgrund des bereits verstrichenen Zeitraums seit Symptombeginn war der Therapieversuch mit Remdesivir nicht indiziert. Zum Zeitpunkt des Krankenhausaufenthaltes des Patienten bestand noch keine Empfehlung zur Therapie mit Tocilizumab nach S3-Leitlinie. Der Patient erhielt durchgehend weiterhin seine therapeutische Antikoagulation mit Apixaban. Seit der Aufnahme zeigte der Patient eine übersteigerte Motivation zur Atemgymnastik, mit selbstinitiierten tiefen und häufigen Atembemühungen. Im Röntgenbild zeigte sich 7 Tage nach Aufnahme eine ovale Hypertransparenz paramediastinal links, die sich in einer CT-Untersuchung als neu aufgetretene große Bulla $(3,5 \times 3,3 \times 10,2 \mathrm{~cm})$ ohne Hinweis auf einen Pneumothorax darstellte ( $\mathbf{\bullet} \mathbf{A b b}$. 2). Zu einer spontanen 
Ruptur der Bulla kam es nicht. Der arterielle Sauerstoffgehalt betrug dauerhaft über $12 \mathrm{ml} / \mathrm{dl}$, dennoch war die respiratorische Insuffizienz trotz Ausschöpfung aller nichtinvasiver Beatmungsmöglichkeiten progredient, sodass am Tag 11 nach Aufnahme die endotracheale Intubation unumgänglich war. Unmittelbar vor Intubation erfolgte ein nichtinvasiver Beatmungsversuch (PEEP 4 mbar; Druckdifferenz 10 mbar, $\mathrm{F}_{1} \mathrm{O}_{2} \quad 100$ Vol.-\%). Die durchgeführte arterielle Blutgasanalyse zeigte die folgenden Parameter: $\mathrm{p}_{\mathrm{a}} \mathrm{O}_{2}$ $57,5 \mathrm{~mm} \mathrm{Hg} ; \mathrm{p}_{\mathrm{a}} \mathrm{CO}_{2} 29,3 \mathrm{~mm} \mathrm{Hg} ; \mathrm{pH} 7,5 ; \mathrm{BE}$ $0,7 \mathrm{mmol} / \mathrm{l}$; Tidalvolumen exspiratorisch $1279 \mathrm{ml}$; Atemfrequenz 29/min; HorowitzIndex $57,5 \mathrm{mmHg}$. Der Oxygenierungsindex des Patienten erfüllte damit die Berlin-Kriterien eines schweren COVID-19assoziierten ARDS. Auch unter der invasiven, druckkontrollierten Beatmung zeigte sich keine Verbesserung des Gasaustausches. Der Patient entwickelte einen rasch progredienten Schock und ein Multiorganversagen (Herz-Kreislauf-Versagen, anurisches Nierenversagen, Laktacidose). Er benötigte hochdosiert kreislaufunterstützende Medikamente (Noradrenalin 2,4 mg/h, Vasopressin 1,5 IE/h, Dobutamin $10 \mathrm{mg} / \mathrm{h}$ ). Die durchgeführte Herzechographie zeigte einen mittelgradig dilatierten rechten Ventrikel mit eingeschränkter Pumpfunktion, einen leicht hypertrophierten und hypovolämischen linken Ventrikel mit guter Pumpfunktion, keine regionalen Wandbewegungsstörungen, keinen Perikarderguss, keine relevanten Klappenvitien. Eine Bauchlage war aufgrund der Instabilität des Patienten nicht möglich, ebenso wenig qualifizierte sich der Patient für eine ECMO-Therapie. Die Prognose musste aufgrund der bereits vor der akuten Verschlechterung manifesten Lungendestruktionen einerseits und des akuten Schockgeschehens andererseits als infaust eingeschätzt werden. Der Patient verstarb noch am 11. Tag nach Aufnahme.

Die Obduktion ergab einen diffusen Alveolarschaden mit viropathischen Veränderungen sowie multiple subpleurale Bullae sowohl der linken Lunge als auch im rechten Lungenunterlappen. Die größte entsprach der im CT dargestellten Bulla im linken Unterlappen, welche mit $11,1 \times 6,0 \times 4,2 \mathrm{~cm}$ beschrieben wurde, ohne Hinweise auf einen Pneumothorax.
Die Todesursache war das respiratorische Versagen bei SARS-CoV-2-Infektion.

\section{Diskussion}

Bullae sind luftgefüllte, dünnwandige Blasen, die durch destruktive Prozesse mit Rarefizierung des Lungenparenchyms entstehen. Sie können primär („,vanishing lung syndrome") oder sekundär im Rahmen eines Lungenemphysems/einer COPD oder anderer Strukturerkrankungen, etwa einer Lungenfibrose, auftreten. Große Bullae können funktionelle Teile der Lunge verdrängen, was zu einer weiteren Verschlechterung des Gasaustausches führt. Eine wesentliche Komplikation ist der Pneumothorax.

Als Folge von COVID-19-Infektionen sind mehrere Fälle von Patienten beschrieben, die unabhängig von pulmonalen Vorerkrankungen Bullae oder konsekutiv Pneumothoraces durch Rupturen entwickelten $[1,6,7,9,11]$. Pathophysiologischer Ausgangspunkt ist eine durch SARS-CoV-2 verursachte diffuse Zerstörung des Alveolargewebes [7], die unter der häufig notwendigen Überdruckbeatmung bei COVID-19-assoziiertem ARDS als „ventilator-induced lung injury" enorm aggravieren kann $[4,5]$. Ob es durch nichtinvasive CPAP-Beatmung zu einer überdruckinduzierten Ausdehnung des durch die COVID-19-Pneumonie geschädigten Lungenparenchyms - und somit zur Bildung von Bullae - kommt, wird ebenfalls diskutiert [1]. In den meisten bislang beschriebenen Fallberichten ging dem Auftreten der Bullae eine (zumindest nichtinvasive) Überdruckbeatmung voraus. Dies stellt aus unserer Sicht ein wesentliches Argument für eine lungenprotektive Beatmung gerade bei COVID19-Erkrankten dar. Des Weiteren muss eine „patient self-inflicted lung injury" ( $P$ SILI) diskutiert werden, bei der es durch patienteneigene Atemarbeit und den dadurch verursachten transpulmonalen Druck zu einem Lungenschaden kommt [10]. Ein wesentlicher Treiber der P-SILI ist der vermehrte Atemantrieb mit erhöhter Atemarbeit und hohen Tidalvolumina. Der dadurch erhöhte transpulmonale Druck kann ein vorbestehendes „capillary leak" - seinerseits am ehesten eine Folge des Lungenschadens durch die SARS-
CoV-2-Infektion - vergrößern und somit das Lungenödem aggravieren [10]. Die NIV-Therapie bei Patienten mit hohem "respiratory drive" kann noch höhere Tidalvolumina zur Folge haben, und die Kontrolle dieser Volumina ist bei Patienten unter NIV-Therapie stark limitiert $[2,3]$. Hohe Tidalvolumina $(>9,5 \mathrm{ml} / \mathrm{kgKG})$ sind dabei prädiktiv für ein NIV-Versagen [3]. Die in diesem Fall beobachteten hohen Tidalvolumina (TV: $1279 \mathrm{ml}$ ) während der nichtinvasiven CPAP-Beatmung unmittelbar vor der Intubation unterstreichen dies. In dem hier vorgestellten Fall ist die Lungenparenchymschädigung mit post mortem nachweisbaren viropathischen Veränderungen auf den schweren Verlauf der COVID-19-Pneumonie mit assoziiertem schwerem ARDS (Horowitz-Index $57,5 \mathrm{~mm} \mathrm{Hg}$ ) und HSV-Superinfektion zurückzuführen, eine Beteiligung im Sinne einer P-SILI als komplizierender Faktor ist zudem wahrscheinlich. Hier entstand die P-SILI jedoch unter reiner Spontanatmung ohne Überdruck unter HFOT, begünstigt durch die exzessive Atemgymnastik mit tiefen und häufigen Atemzügen seitens des Patienten. Hervorzuheben ist zudem die äußerst rapide Entwicklung dieses destruktiven Lungenprozesses, der während des akuten intensivmedizinischen Aufenthaltes, innerhalb von 7 Tagen nach Aufnahme auf die Intensivstation, auftrat.

In der Zukunft muss untersucht werden, ob durch hochprotektive und frühzeitige Ventilation unter Suppression des spontanen Atemantriebs mit minimaler Bewegung des Lungenparenchyms $\left(\mathrm{V}_{T} \leq 4 \mathrm{ml}\right.$ $\mathrm{kg}^{-1}$ und Bauchlagerung) und verminderter Fluktuation des transpulmonalen Drucks die P-SILI verhindert und so Lungenstrukturschäden vermieden werden können.

Zusammengefasst unterstreicht der geschilderte Fall, dass es bei einer COVID19-Pneumonie mit assoziiertem schwerem ARDS bei Patienten ohne pulmonale Grunderkrankung zu strukturellen Umbauprozessen mit der Bildung von Bullae kommen kann. Die Möglichkeit einer Ruptur dieser Bullae wurde bereits in Einzelfällen beschrieben und kann spontan oder durch Überdruckbeatmung auftreten $[1,6,7,9,11]$. Bei einer respiratorischen Verschlechterung dieser Patienten sollte differenzialdiagnostisch demnach eine 
Verdrängung des funktionellen Lungengewebes durch eine Riesenbulla bzw. ein Pneumothorax durch deren Ruptur bedacht werden.

\section{Fazit für die Praxis}

- Schwere Verläufe der COVID-19-Pneumonie können zu Lungenstrukturläsionen mit der Ausbildung von Bullae führen.

- Die CT-Bildgebung ermöglicht die sichere Diagnosestellung.

- Der Zusammenhang zwischen nichtinvasiver CPAP-Beatmung und der Entwicklung von Bullae und Pneumothoraces bei COVID-19-Patienten sollte zukünftig untersucht werden.

- Auch bei Patienten mit Spontanatmung und HFOT können Lungenparenchymschäden entstehen, die durch die P-SILI aggraviert werden.

\section{Korrespondenzadresse}

\section{PD Dr. med. Daniela Hauer}

Intensivstation ANIS 5, Klinik für Anaesthesiologie, Klinikum der Universität München, LMU München

Nussbaumstraße 20, 80336 München,

Deutschland

daniela.hauer@med.uni-muenchen.de

Funding. Open Access funding enabled and organized by Projekt DEAL.

\section{Einhaltung ethischer Richtlinien}

Interessenkonflikt. N. Moellhoff, P. Groene, L. Ney und D. Hauer geben an, dass kein Interessenkonflikt besteht.

Für diesen Beitrag wurden von den Autoren keine Studien an Menschen oder Tieren durchgeführt. Für die aufgeführten Studien gelten die jeweils dort angegebenen ethischen Richtlinien. Für Bildmaterial oder anderweitige Angaben innerhalb des Manuskripts, über die Patienten zu identifizieren sind, liegt von ihnen und/oder ihren gesetzlichen Vertretern eine schriftliche Einwilligung vor.

Open Access. Dieser Artikel wird unter der Creative Commons Namensnennung 4.0 International Lizenz veröffentlicht, welche die Nutzung, Vervielfältigung, Bearbeitung, Verbreitung und Wiedergabe in jeglichem Medium und Format erlaubt, sofern Sie den/die ursprünglichen Autor(en) und die Quelle ordnungsgemäß nennen, einen Link zur Creative Commons Lizenz beifügen und angeben, ob Änderungen vorgenommen wurden.

Die in diesem Artikel enthaltenen Bilder und sonstiges Drittmaterial unterliegen ebenfalls der genannten Creative Commons Lizenz, sofern sich aus der Abbildungslegende nichts anderes ergibt. Sofern das betreffende Material nicht unter der genannten Creative

\section{Development of a giant bulla under spontaneous breathing by self- inflicted lung injury in a patient with COVID-19 pneumonia}

The outbreak of SARS-CoV-2 and the associated COVID-19 pandemic pose major challenges to healthcare systems worldwide. New data on diagnosis, clinical presentation and treatment of the disease are published on a daily basis. This case report describes the fatal course of severe COVID-19 pneumonia in an 81-year-old patient with no previous pulmonary disease who developed a giant bulla during noninvasive high-flow oxygen therapy. Virus-induced diffuse destruction of alveolar tissue or patient self-inflicted lung injury (P-SILI) are discussed as possible pathomechanisms. Future studies must determine whether lung-protective mechanical ventilation with high levels of sedation and paralysis to suppress spontaneous respiratory drive and to reduce transpulmonary pressure can prevent structural lung damage induced both by the virus and P-SILI in COVID-19 patients with ARDS.

\section{Keywords}

COVID-19 · ARDS · Bulla · High-flow therapy · Patient self-inflicted lung injury

Commons Lizenz steht und die betreffende Handlung nicht nach gesetzlichen Vorschriften erlaubt ist, ist für die oben aufgeführten Weiterverwendungen des $\mathrm{Ma}$ terials die Einwilligung des jeweiligen Rechteinhabers einzuholen.

Weitere Details zur Lizenz entnehmen Sie bitte der Lizenzinformation auf http://creativecommons.org/ licenses/by/4.0/deed.de.

\section{Literatur}

1. Berhane S, Tabor A, Sahu A et al (2020) Development of bullous lung disease in a patient with severe COVID-19 pneumonitis. BMJ Case Rep. https://doi.org/10.1136/bcr-2020-237455

2. Brochard L, Slutsky A, Pesenti A (2017) Mechanical ventilation to minimize progression of lung injury in acute respiratory failure. Am J Respir Crit Care Med 195:438-442

3. Carteaux G, Millan-Guilarte T, DeProst Netal (2016) Failure of noninvasive ventilation for de novo acute hypoxemic respiratory failure: role of tidal volume. Crit Care Med 44:282-290

4. Cruz FF, Ball L, Rocco PRM et al (2018) Ventilatorinduced lung injury during controlled ventilation in patients with acute respiratory distress syndrome: less is probably better. Expert Rev Respir Med 12:403-414

5. Deliwala SS, Ponnapalli A, Seedahmed Eet al (2020) A 29-year-old male with a fatal case of COVID-19 acute respiratory distress syndrome (CARDS) and ventilator-induced lung injury (VILI). Am J Case Rep 21:e926136

6. Fan Q, Pan F, Yang L (2020) Spontaneous pneumothorax and subpleural bullae in a patient with COVID-19: a 92-day observation. Eur J Cardiothorac Surg 58:858-860

7. Janssen ML, Van Manen MJG, Cretier SE et al (2020) Pneumothorax in patients with prior or current COVID-19 pneumonia. Respir Med Case Rep 31:101187

8. Kluge $S$, Janssens U, Welte $T$ et al (2020) Recommendations for treatment of critically ill patients with COVID-19: version 3 S1 guideline. Anaesthesist. https://doi.org/10.1007/s00101020-00833-3
9. Sun R, Liu H, Wang X (2020) Mediastinal emphysema, giant bulla, and pneumothorax developed during the course of COVID-19 pneumonia. Korean JRadiol 21:541-544

10. Windisch W, Weber-Carstens S, Kluge S et al (2020) Invasive and non-invasive ventilation in patients with COVID-19. Dtsch Arztebl Int 117:528-533

11. Yasukawa K, Vamadevan A, Rollins R (2020) Bulla formation and tension pneumothorax in a patient with COVID-19. Am J Trop Med Hyg 103:943-944 